\section{X-ray pulsars}

\section{Spin-up changes to spin-down}

\author{
N. E. White
}

Elsewhere in this issue, K. Makishima $e t$ al. (Nature 333, 746-748; 1988) report that the spin-rate of the X-ray pulsar $\mathrm{GX} 1+4$, which they have been observing with the $\mathrm{X}$-ray satellite Ginga, is now decreasing whereas previous observations had shown that it was increasing. This fits neither of the two behaviours observed in the other 25 known X-ray pulsars: continuous spinup lasting for decades or alternate spinup and spin-down on timescales of hours to months. The unusual behaviour of $\mathrm{GX} 1+4$ is probably related to a change in its interaction with material captured from a nearby companion star.

$\mathrm{X}$-ray pulsars are spinning neutron stars capturing material from a binary companion. The strong magnetic field of the neutron star funnels the accreted material down onto its poles where the gravitational energy is released as X-rays. The rotation of this hotspot sweeps the X-rays like the beam from a lighthouse, causing the characteristic pulses observed. Spin-up is expected because the accretion flow contains angular momentum which will be added to that of the neutron star. Spindown is poorly understood.

$\mathrm{X}$-ray pulsars powered by the capture of wind from a young luminous $\mathrm{O}-$ or B-star companion switch between intervals of spin-up and spin-down on timescales of hours to months. Two-dimensional hydrodynamic calculations show that the flow pattern of an OB star's wind to a neutron star is not steady, and can reverse direction. This causes the pulsar to be spun up and spun down in the same direction as the accretion disk. In other cases where the companion fills or nearly fills its Roche lobe (the volume beyond which it sheds gas passively onto the neutron star) pulsars spin up continuously on timescales of up to 50 years, because a steady gas stream feeds the accretion disk.

An X-ray pulsar can also change from spin-up to spin-down because of increased drag on the neutron star's magnetosphere by an accretion disk. This occurs when the co-rotation velocity of material threaded onto the magnetic field lines is comparable to the keplerian (stable-orbital) velocity. If the accretion rate decreases, the radius at which the magnetic field disrupts the accretion disk increases until the keplerian velocity there equals the spin velocity. An appreciable spin-down torque is then exerted even though accretion continues (Ghosh, P. \& Lamb, F. K. Astrophys. J. 232, 296-316; 1979). At even lower accretion rates, when the co-rotation velocity exceeds the keplerian velocity, accretion onto the neutron star ceases because of centrifugal forces.

The accretion rate in the $\mathrm{GX} 1+4$ system may have decreased just to the critical point where spin-down can occur although accretion continues. The keplerian period in the accretion disk of 110 seconds corresponding to the spin period of the neutron star occurs at a radius of about 40,000 kilometres. A magnetic field of about $10^{14}$ gauss is required to disrupt the disk at this radius, much more than the field of about $10^{12}$ gauss usually associated with pulsars.

Makishima et al. dislike invoking such a high magnetic field and prefer the model used to explain the episodes of spin-up and spin-down of X-ray pulsars orbiting $\mathrm{OB}$ stars. GX1+4 is unique among X-ray pulsars with late-type companions. Its binary companion is an M6III star which could have a substantial wind causing a significant torque on the neutron star. The problem for this model is that the wind of an M6III star is a hundred times slower than that of an OB star. The captured specific angular momentum scales as the fourth power of the velocity and for an M-star companion, a large stable disk rotating in the same sense as the binary will form and is unlikely to reverse direction.

In my view, the model that requires the magnetic field to be about $10^{14}$ gauss is more likely. This is rather high when compared with the range found from radio pulsars which has a maximum of about $10^{1.3}$ gauss, but is not excluded on physical grounds. In fact it has been speculated that the cut off at the high end of the magnetic field distribution of radio pulsars indicates that the elusive radio pulse mechanism only operates over a selective magnetic field range (Radhakrishna, V. Contemp. Phys. 23, 207-231; 1982). The $\mathrm{X}$-ray pulsars are pulsed by viewing a rotating beam of X-rays from the magnetic poles, a mechanism not strongly dependent on the strength of the magnetic field.

As noted by Makishima et al. in this issue, this observation of spin-down from $\mathrm{GX} 1+4$ neatly confirms the view that the rapid spin-up timescale of about 50 years observed in pulsars such as $\mathrm{GX} 1+4$ cannot be sustained and that these pulsars must also undergo extended intervals of spindown. The rate of spin-down of $\mathrm{GX} 1+4$ will allow a test of spin-down models and also provide a more soundly based estimate of the number of dormant X-ray pulsars in the Galaxy.

N. E. White is at the EXOSAT Observatory. Space Science Department of ESA, $2200 A G$ Noordwijk, The Netherlands.

\section{Daedalus}

\section{Psychic chaos}

THE evidence for paranormal effects like psychokinesis is very anecdotal and statistical. Most scientists discount it, arguing that psychic forces must be vanishingly small to have evaded detection and measurement all these years, and that in any case a world governed by the laws of physics has no room for them. Daedalus now points out that in 'chaotic' systems the grip of physics is severely weakened.

Chaos is a hot topic these days, and can govern even classically deterministic objects like pendulums. Two different starting conditions of such a chaotic pendulum, even if arbitrarily close together, will rapidly evolve into quite different patterns of swing. So, says Daedalus, the final state of such a pendulum must ultimately be influenced by initial perturbations smaller than its limiting Heisenberg uncertainty. Here is a region, closed to physics even in theory, where extremely tiny psychic effects could be 'amplified' into final dominance.

The largest and most important chaotic system is, of course, the weather. In temperate regions like western Europe, the weather is generated by the continual self-amplification of turbulence between conflicting oceanic winds. On every timeand distance-scale, random fluctuations appear and grow. Current forecasts reach a week ahead; with detailed knowledge of much smaller atmospheric features, two weeks might be feasible; but six weeks ahead the controlling perturbations are below the Heisenberg limit and beyond physics. So, says Daedalus, it may be entirely rational to pray for rain, provided you do it six weeks in advance.

To test this bold speculation, DREADCO's statisticians are studying the weather records of the past century or so. Have events like harvest times and national holidays, the subject of fervent and longrange public wishing, been sunnier than you would expect? If so, Daedalus will try to discover how many people are needed to produce a useful psychic effect. Traditional psychic experiments on single individuals are always infuriatingly inconclusive; a mass experiment should multiply any tiny effects up to convincing reproducibility. So DREADCO's engineers are building a 'chaotic dice' machine in which subHeisenberg effects can be amplified up to dominance in a minute or so of tumbling. Daedalus will then use it to play "Wish for the number" games in front of a public meeting or, better, a television audience. If a big audience can indeed control a chaotic dice by mass wishing, psychic research will be revolutionized. Meanwhile, it will soon be time to start dreaming of a white Christmas.

David Jones 